\title{
Mice with half a mind
}

\section{Nigel Holder and Malcolm Maden}

THE effect of the putative morphogen retinoic acid on the developing vertebrate nervous system has been brought into sharper focus by the paper by Marshall et al. on page 737 of this issue ${ }^{1}$. Using a line of transgenic mice in which a reporter gene is linked to the promoter for one of the Hox genes implicated in brain development, they show clearly that retinoic acid treatment at the gastrula stage leads to a transformation in the rhombomeres of the anterior hindbrain region in which rhombomeres 2 and 3 are respecified as 4 and 5 respectively.

Retinoic acid has attracted much attention over the past few years because of the compound's remarkable effects on the tissue pattern of developing limbs and the discovery of the burgeoning family of nuclear retinoid receptors. Less attention has been paid to the effects of retinoic acid on the developing vertebrate nervous system ${ }^{2}$. Most notable of these effects is the truncation of anterior head and brain structures and abnormal formation of the hindbrain, highlighted in 1989 by Durston et al. ${ }^{3}$, after treatment of Xenopus gastrulae with retinoic acid.

Durston and colleagues suggested that the abnormal phenotype was caused by respecification of anterior brain structures - forebrain and possibly anterior midbrain - into more posterior hindbrain. This neatly unified the action of retinoic acid on the patterning process within the embryo, as it also posteriorizes limb cells. But Marshall et al.'s results confirm work carried out by various groups over the past three years which has pinpointed the anterior hindbrain as the site of the retinoic acid effect. The respecification of anterior hindbrain is the common and predictable effect of exposure of gastrulae of various vertebrates - mouse, chick, Xenopus and zebrafish - to a short burst of retinoic acid ${ }^{4-7}$.

This effect on the hindbrain tied the teratology to another action of retinoic acid: its regulation of Hox gene complexes in a time- and dose-specific manner ${ }^{8,9}$. These are the very genes that show limits of anterior expression patterns among the segmental units of the hindbrain ${ }^{10}$. This link prompted several groups to examine the expression pattern of relevant Hox genes in the anterior hindbrain of embryos treated with retinoic acid. Hox-2.9, which is normally expressed in the hindbrain only in rhombomere 4 , is overexpressed more anteriorly $\mathrm{y}^{4,11}$

Here the story has rested up until now. The abnormal expression of Hox2.9 and other segmentally restricted genes such as Krox-20 4,6,11 provided a link to the altered morphology but could not be clearly related to the acquisition of segmental identity for each of the rhombomeres. Marshall et al. carried out the same basic experiment as before: embryos in utero were exposed to retinoic acid at the gastrula stage, but two new features of their experimental design have revealed these striking new results. First, they used a line of transgenic mice carrying a lac $Z$ reporter gene under the control of the Hox-2.9 promoter to visualize the expression of the Hox-2.9 gene in experimental animals. Second, the embryos were examined at stages later than in previous studies.

At the early stages of hindbrain formation in these embryos, Hox-2.9 expression shows the predicted anterior expansion; however, in embryos allowed to develop a little longer this homogeneous expanded anterior region resolves into two clear stripes of Hox-2.9. What was an ill-defined anterior hindbrain seems to produce two rhombomere $4 \mathrm{~s}$ separated by a clear rhombomere width. Probing with Krox20 , which normally expresses in rhombomeres 3 and 5 , strongly suggests that this rhombomere is a second rhombomere 5, giving a pattern of a 4-5-4-5 repeat in the anterior region followed by the normal posterior rhombomere pattern. Anatomical analysis using backfills of fluorescent dye to label identifiable motor nuclei confirms the interpretation derived from the molecular markers.

The cells expressing Hox-2.9 abnormally in the anterior hindbrain must interact in some way to cause the formation of a clear anatomical unit. Whatever the mechanism proves to be, it is likely to tell us something direct about the normal formation of the hindbrain rhombomere pattern and the role that Hox genes play in establishing segmental identity. The other crucial finding from these studies is that downstream retinoic acid effects lead to a clear respecification of anterior rhombomeres to posterior rhombomeres. The patterns generated in the current experiments appear to replace rhombomeres 2 and 3 with 4 and 5 . No additional segmental units are described, although it is unclear what is going on with rhombomere 1 and the hindbrain-midbrain border region. Marshall et al. also show that respecification of rhombomeres leads to respecification of neural crest derivatives associated with each segment. The retinoic acid therefore alters the anatomy of the head as a consequence of altered behaviour of respecified crest cells, reinforcing the view that the Hox code is crucial for the patterning of the head as a whole, not simply for the central nervous system.

A major question remains about these and related experiments. What is the primary effect of retinoic acid at the gastrula stage? The identification of this effect will be instructive because it may reveal the normal chain of command of interactions leading to the control of Hox-2.9 expression in rhombomere 4 and to the expression of other regulatory genes in the hindbrain. It is important to know, for example, if the retinoic acid effect on Hox-2.9 is direct. In the mouse, this gene and its paralogue in the Hox-1 cluster, Hox-1.6, are expressed during gastrulation, as are their homologues in other species ${ }^{12-14}$. Interestingly, the Hox-1.6 gene has recently been shown to contain a retinoic acid $\beta$ receptor response element in the $3^{\prime}$ region $^{15}$, although it is not yet known whether this is functional. It may be the case, then, that the primary retinoic acid effect is on expression of the Hox-1.6 gene, which then secondarily regulates Hox-2.9 expression. Consistent with this notion is the intriguing observation that the phenotype of the Hox-1.6 mutant mouse produced by homologous recombination ${ }^{16,17}$, is grossly similar to the phenotype generated by retinoic acid in vertebrate embryos. Both generate clear-cut alterations to the development of the hindbrain region of the head.

Nigel Holder and Michael Maden are at the Developmental Biology Research Centre, Randall Institute, King's College London, 26-29 Drury Lane, London WC2B 5RL, UK.

\footnotetext{
1. Marshall, H., Nonchev, S., Sham, M. H., Muchamore, I., Lumsden, A., \& Krumlauf, R. Nature 360, 737-741 (1992).

2. Maden, M. \& Holder, N. BioEssays 14, 431-438 (1992).

3. Durston, A. J. et al. Nature 340, 140-144 (1989).

4. Morriss-Kaye, G., Murphy, P., Hill, R. \& Davidson, D. EMBO J. 10, 2985-2995 (1991).

. Sundin, O. \& Eichele, G. Development 114, 841-852 (1992).

6. Papalopulu, N. et al. Development 113, 1145-1158 (1991).

7. Holder, N. \& Hitl, J. Development 113, 1159-1170 (1991).

8. Simeone, A. et al. Nature 346, 763-766 (1990).

. Papalopulu, N., Lovell-Badge, R. \& Krumlauf, R. Nucl. Acid. Res. 19, 5497-5506 (1991).

10. Wilkinson, D. \& Krumlauf, R. Trends Neurosci. 13 335-339 (1990)

11. Conton, R. \& Rossant, J. Development 116, 357-368 (1992).

12. Frohman, M., Boyle, M. \& Martin, G. Development 110. 589-607 (1990)

13. Sive, H. \& Cheng, P. Genes Dev. 5, 1321-1332 (1991).

14. Sundin, O., Busse, H., Gudas, L. \& Eichele, G. Development 108, 47-58 (1990).

15. Langston, A. W. \& Gudas, L. Mech. Dev. 38, 217-228 (1992).

16. Lufkin, T., Dierich, A., LeMeur, M., Mark, M. \& Chambon, P. Cell 66, 1105-1119 (1991).

17. Chisaka, O. \& Capecchi, M. Nature 350, 473-479 (1991)
} 